\title{
Diabetes mellitus type 2 does not influence carotid stiffness in patients on maintenance hemodialysis
}

Konrad Rekucki ${ }^{1}$, Agnieszka Sławuta ${ }^{2}$, Marta Obremska ${ }^{3}$, Katarzyna Madziarska $^{4}$

${ }^{1}$ Department of Cardiology, T. Marciniak Specialist Lower Silesian Hospital, Wrocław, Poland

${ }^{2}$ Department of Internal and Occupational Diseases, Hypertension and Clinical Oncology, Wroclaw Medical University, Wrocław, Poland

${ }^{3}$ Department of Preclinical Research, Wroclaw Medical University, Wrocław, Poland

${ }^{4}$ Department of Nephrology and Transplantation Medicine, Wroclaw Medical University, Wrocław, Poland

Abstract

Patients on maintenance hemodialysis are a group with high cardiovascular risk, characterized by high arterial stiffness, which is considered a novel cardiovascular risk factor. Diabetes mellitus is both one of the leading causes of end-stage renal disease and a determinant of poor outcome in this group. The aim of the study was to examine carotid stiffness with high resolution echo-tracking in order to assess the influence of diabetes mellitus on arterial stiffness in this group.

Ninety patients (47 F; $43 \mathrm{M}$ ) with end-stage renal disease on maintenance hemodialysis were divided into two subgroups: diabetic and nondiabetic ( 37 and 53 patients respectively). They underwent clinical examination, laboratory tests, and ultrasonographic carotid stiffness assessment both before and after hemodialysis. Local arterial stiffness parameters $\beta, E p, A C$, and PWV $\beta$ were calculated. Patient survival was assessed after a 58-month-long follow-up.

During the 58-month period 25 of these diabetic patients died, as did 18 non-diabetic patients. Diabetes mellitus was a risk factor for overall mortality among the group of hemodialysed patients. Patients who died from non-cardiovascular causes significantly more often suffered from diabetes mellitus than survivors. There were no statistically significant differences in local arterial stiffness between the groups.

Local arterial stiffness in hemodialysed patients, assessed with high resolution echo-tracking, is not influenced by the presence of diabetes.

Keywords

Chronic kidney disease $\bullet$ echo-tracking $\bullet$ diabetes mellitus $\bullet$ hemodialysis $\bullet$ arterial stiffness

Received: 08.03.2021, Accepted: 17.08.2021

\section{INTRODUCTION}

End-stage renal disease patients are a group with high cardiovascular mortality, which is further worsened by the coexistence of diabetes [1]. As diabetic nephropathy is the leading cause of end-stage renal disease, the presence of both issues is a common clinical condition [2]. While diabetes mellitus alone is also a well-established risk factor for poor cardiovascular outcome, the search for better risk prediction methods is the key to improvement of survival in these patients. The importance of end-stage renal disease exceeds by far its clinical implications. Maintenance hemodialysis is a life-altering process for every patient. Moreover, the endstage renal disease population accounts for much higher rates of medical spending in healthcare systems than that of the general population [3].

The role of arterial stiffness as a cardiovascular risk factor is already established in clinical practice $[4,5]$. It is a property of the arterial tree resulting from the ratio of elastic to collagen fibers in the arterial wall [6]. Arterial rigidity progresses with age and accelerates in such diseases as hypertension, diabetes mellitus, and chronic kidney disease $[7,8]$. High arterial stiffness results in increased risk of stroke, myocardial infarction, and overall cardiovascular mortality $[9,10]$. The progression of chronic kidney disease leads to premature vascular aging, including many local vascular and general mechanisms. Chronic inflammation with high levels of proinflammatory cytokines, oxidative stress and imbalance 
between inducers and inhibitors of vascular calcification, are the main mechanisms leading to progression of arterial stiffness in this group [11]. Diabetes mellitus similarly leads to arterial wall remodeling through enhancement of nonenzymatic glycation of proteins, with covalent irreversible cross-linking of collagen and creation of advanced glycation end-products (AGEs) [12, 13].

The complexity of the vascular system leads to different methods of assessing arterial stiffness. Depending on the method, regional or local arterial stiffness can be examined [6]. High-resolution echo-tracking is a method of establishing local arterial stiffness, and is usually obtained at the site of the common carotid artery, in contrast to pulse wave velocity, which is a parameter of regional arterial stiffness. There has been a growing interest in the use of high-resolution echotracking and the utility of carotid stiffness assessment in the end-stage renal disease population $[14,15,16]$.

\section{THE AIM}

Our goal was to examine carotid stiffness by means of highresolution echo-tracking in hemodialyzed patients in order to assess the influence of the presence of diabetes mellitus on arterial stiffness in this group.

\section{MATERIAL AND METHODS}

\section{Study population}

The study was performed in 90 patients (female 47 ; male 43 ), aged $64+/-12$, treated with maintenance hemodialysis in a University Hospital. The patients were median 31.6 [9.5-83.0] months on the renal replacement therapy at time when they were included in the study. The study group was primarily divided into two subgroups: diabetic and nondiabetic. The study group was collected in January 2015. We excluded the patients with overt infection. Carotid rigidity was evaluated directly with the use of ultrasonographic echotracking before and after hemodialysis section and local arterial stiffness parameters were calculated. Patient survival was assessed in November 2019, concluding a 58-monthlong follow-up. Baseline characteristics of subjects such as gender, age, race, body mass index (BMI), duration of dialysis, and adequacy of dialysis $(\mathrm{Kt} / \mathrm{V})$ were assessed at the study onset. The laboratory parameters were obtained based on blood samples collected prior to initiation of the hemodialysis session. Hypertension was treated with angiotensin converting enzyme (ACE) inhibitors, angiotensin receptor blockers (ARBs), calcium channel blockers, betablockers and alpha-blockers. All patients were treated with intravenous iron supplementation and erythropoiesis stimulating agents (ESAs) according to standards. In all subjects native arteriovenous fistula was the vascular access used for the dialysis. Data on cardiovascular morbidity and causes of end-stage renal disease were collected based on anamnesis and hospital records of patients.

\section{Study group examination}

Laboratory tests took place at the study onset in the Central Hospital laboratory as a part of routine medical care. Transthoracic echocardiographic examination was performed in all subjects. All patients underwent echo-tracking examination of the common carotid artery both before and after a single hemodialysis session at the beginning of the observation period, preceded by measuring blood pressure on the brachial artery.

\section{Assessment of local arterial stiffness}

Images were obtained with an Aloka Alpha-6 ultrasonograph equipped with an integrated and automated Doppler and high-resolution echo-tracking system, using a linear probe 15 minutes before and after hemodialysis. The examination was performed by two experienced ultrasonographers. For performing echo-tracking measurements we established an ultrasonographic view of the common carotid artery (opposite to the arteriovenous shunt) in its longitudinal axis with a clear visualization of the anterior and posterior walls. Subsequently, the intima-media complex $1-2 \mathrm{~cm}$ below the carotid bifurcation was established and echo-tracking samples were set at the end of the intima for continuous detection of the arterial wall movement. As a result, a waveform was obtained to show a graphical presentation of change in the diameter of the artery.

A representative waveform was created automatically after recording of three to five proper evolutions. To calculate local arterial stiffness parameters, simultaneous ECG monitoring has been performed using standard electrocardiographic limb leads; blood pressure was measured in supine position directly before the echo-tracking examination over the brachial artery opposite to the arteriovenous shunt.

High-resolution echo-tracking allowed the calculation of the following parameters:

Beta stiffness index ( $\beta$ ):

$\beta=\ln (P s / P d) /[(D s-D d) / D d]$

One-point pulse wave velocity $(P W V \beta)$ :

$P W V \beta=\sqrt{ }\left(B^{*} P / 2^{*} r\right)$

Arterial compliance (AC):

$A C=\pi(D s \times D s-D d \times D d) /[4 \times(P s-P d)]$ 
Young's modulus, Epsilon (Ep):

$E p=(P s-P d) /[(D s-D d) / D d]$

The abbreviations used in the aforementioned equations stand for: In — the natural logarithm; Ps — systolic blood pressure; Pd - diastolic blood pressure; Ds - diameter of the artery in systole; Dd — diameter of the artery in systole; $\mathrm{P}$ - diastolic blood pressure; $r$ - blood density $\left(1.050 \mathrm{~kg} / \mathrm{m}^{3}\right)$.

The study was approved by the Institutional Ethics Committee (Consent No 342).

\section{Statistical analysis}

Statistical analysis was performed using the Statistica 12 (TIBICO Inc., USA) software under license of the Wroclaw Medical University, Poland. Discrete variables were presented as numbers and percentages and compared with a chi-squared test. Continuous variables were presented as medians and interquartile ranges (IQR) because most of them did not have normal distribution; these were compared with the Mann Whitney $U$ test. Normality of data distribution was assessed with the Shapiro-Wilk test. $P$ value less than 0.05 was regarded as significant.

\section{RESULTS}

The baseline clinical characteristics of the study subgroups regarding the etiology of chronic kidney disease and cardiovascular co-morbidity are presented in Tables 1 and 2.

The most common etiology of chronic kidney disease in the diabetic group was hypertensive kidney disease, while in the non-diabetic group the primary cause was chronic glomerular disease. In terms of cardiovascular morbidity, coronary artery disease was more common in the diabetic group. In this group more patients underwent surgical revascularization as well. As for hypertension, myocardial infarction, stroke, and history of percutaneous coronary intervention, there was no significant difference between the groups. We found no difference between the groups in the occurrence of cancer.

The comparisons of basic demographic, clinical, hemodialysis, and laboratory data between the diabetic and non-diabetic groups are presented in Table 3.

Subjects with diabetes mellitus tended to be older than non-diabetic patients and were predominately female (59.5\%). Diabetic patients had significantly lower pre-dialysis creatinine concentration, which in combination with higher body weight (not statistically different, but within overweight values in diabetic patients and normal values in non-diabetic patients) could indicate lower muscle mass and higher fat tissue mass. The two groups did not differ in terms of left
Table 1. Chronic kidney disease etiology

\begin{tabular}{cccc}
\hline & $\begin{array}{c}\text { Diabetic } \\
\mathbf{N = 3 7} \\
\mathbf{n}(\%)\end{array}$ & $\begin{array}{c}\text { Nondiabetic } \\
\mathbf{N = 5 3} \\
\mathbf{n}(\%)\end{array}$ & $\mathbf{p}$ \\
\hline Hypertensive nephropathy & $22(59.5)$ & $11(20.8)$ & $<0.001$ \\
Diabetic nephropathy & $8(21.6)$ & $0(0)$ & $<0.001$ \\
Chronic glomerular disease & $3(8.1)$ & $21(39.6)$ & $<0.001$ \\
Polycystic kidney disease & $1(2.7)$ & $7(13.2)$ & 0.18 \\
Interstitial nephropathy & $2(5.4)$ & $8(15.1)$ & 0.12 \\
Other & $1(2.7)$ & $7(13.2)$ & 0.08 \\
\hline
\end{tabular}

Table 2. Cardiovascular co-morbidity

\begin{tabular}{cccc}
\hline & $\begin{array}{c}\text { Diabetic } \\
\mathbf{N = 3 7} \\
\mathbf{n}(\%)\end{array}$ & $\begin{array}{c}\text { Non-diabetic } \\
\mathbf{N}=53 \\
\mathbf{n}(\%)\end{array}$ & $\mathbf{p}$ \\
\hline Arterial hypertension & $31(83.8)$ & $38(71.7)$ & 0.18 \\
Myocardial infarction & $8(21.6)$ & $7(13.2)$ & 0.29 \\
Coronary artery disease & $21(56.8)$ & $17(32.1)$ & 0.02 \\
Coronary artery bypass graft & $6(16.2)$ & $1(1.9)$ & 0.01 \\
Percutaneous cardiac intervention & $9(24.3)$ & $7(13.2)$ & 0.17 \\
Stroke & $8(21.6)$ & $4(7.6)$ & 0.053 \\
\hline
\end{tabular}

ventricle ejection fraction, hemoglobin, lipidogram, C-reactive protein, urea before $H D$, urea after $H D$, and creatinine after HD. The obvious difference between the subgroups was fasting glucose concentration.

High-resolution echo-tracking examination has shown no statistically significant differences between the diabetic and non-diabetic groups in terms of arterial stiffness, both before and after hemodialysis. The two groups did not differ with regard to blood pressure and heart rate. The echo-tracking parameters also did not differ significantly in diabetic and non-diabetic groups younger and older than 65 years of age. The respective values are depicted in Table 4.

During the 58-month follow-up 25 out of 37 diabetic patients died, while 18 out of 53 non-diabetic patients died. Diabetes mellitus was a risk factor for overall mortality among the group of hemodialyzed patients. Patients who died from non-cardiovascular causes suffered from diabetes mellitus significantly more often than survivors, while there was no such relation in patients who suffered cardiovascular death. Among the non-cardiovascular reasons for deaths, the most common causes were sepsis and malignancy. The comparison of diabetic and non-diabetic patients (survivors and non-survivors) depending on the cause of death is presented in Table 5. 
Table 3. Basic demographic, clinical, hemodialysis, and laboratory data in the diabetic and non-diabetic groups.

\begin{tabular}{|c|c|c|c|}
\hline & $\begin{array}{c}\text { Diabetic } \\
\mathrm{N}=37 \\
\text { median (IQR) }\end{array}$ & $\begin{array}{c}\text { Non diabetic } \\
\mathrm{N}=53 \\
\text { median (IQR) }\end{array}$ & $\mathbf{p}$ \\
\hline Age [years] & $67[59-76$ ] & $62[46-76]$ & 0.13 \\
\hline Male gender, $\mathrm{n}(\%)$ & 15 [40.55] & 28 [52.83] & 0.25 \\
\hline Female gender, $\mathrm{n}(\%)$ & $22[59.45]$ & $25[47.17]$ & 0.25 \\
\hline Weight before hemodialysis $[\mathrm{kg}]$ & $74.4[61.2-91.3]$ & $67.7[60.9-80.3]$ & 0.40 \\
\hline Body mass index [kg/m2] & $27.3[22.5-30.8]$ & $24.6[23.0-27.6]$ & 0.06 \\
\hline Left ventricular ejection fraction [\%] & 60 [50-65] & $60[50-60]$ & 0.94 \\
\hline Duration of HD therapy [months] & $31.0[10-58.5]$ & $33.8[9.5-95.0]$ & 0.68 \\
\hline Ultrafiltration $[L]$ & $1.9[1.3-2.5]$ & $1.8[1.0-2.0]$ & 0.20 \\
\hline Adequacy of hemodialysis [kT/V] & $1.28[1.11-1.50]$ & $1.40[1.10-1.65]$ & 0.22 \\
\hline SBP before $\mathrm{HD}[\mathrm{mmHg}]$ & $145[120-150]$ & $145[130-160]$ & 0.43 \\
\hline SBP after HD [mmHg] & $140[125-160]$ & $130[120-158]$ & 0.56 \\
\hline DBP before $\mathrm{HD}[\mathrm{mmHg}]$ & $70[60-80]$ & 80 [70-90] & 0.019 \\
\hline DBP after $\mathrm{HD}[\mathrm{mmHg}]$ & $73[65-80]$ & $80[70-89.5]$ & 0.27 \\
\hline $\mathrm{HR}$ before $\mathrm{HD}[\mathrm{mmHg}]$ & 75 [61-86] & 72 [67-79] & 0.72 \\
\hline $\mathrm{HR}$ after HD [mmHg] & $72.5[63-83]$ & 80 [65-84] & 0.55 \\
\hline Urea before $\mathrm{HD}[\mathrm{mg} / \mathrm{dl}]$ & 114 [92-132] & 117 [99-137] & 0.65 \\
\hline Urea after HD [mg/dl] & 36 [27-47] & 35 [26-46] & 0.68 \\
\hline Creatinine before HD [mg/dl] & $6.46[3.35-7.95]$ & $8.04[5.57-9.65]$ & 0.038 \\
\hline Creatinine after HD [mg/dl] & $2.73[1.99-3.81]$ & $2.73[2.15-4.26]$ & 0.44 \\
\hline Hemoglobin [g/dl] & $10.8[9.4-11.4]$ & $10.4[9.5-12.1]$ & 0.95 \\
\hline Total cholesterol [mg/dl] & 167 [142-209] & 171 [146-209] & 0.76 \\
\hline High-density lipoprotein [mg/dl] & $40[34-46]$ & $42[36-48]$ & 0.58 \\
\hline Low-density lipoprotein [mg/dl] & 92 [69-129] & 95 [76-120] & 0.83 \\
\hline Triglycerides [mg/dl] & 165 [119-263] & $146[95-221]$ & 0.13 \\
\hline C-reactive protein $[\mathrm{mg} / \mathrm{l}]$ & $5.63[3.03-23.07]$ & $8.48[3.2-19.5]$ & 0.69 \\
\hline Glucose [mg/dl] & 153 [118-203] & 98 [90-112] & $<0.001$ \\
\hline
\end{tabular}

SBP - systolic blood pressure; DBP - diastolic blood pressure; HR- heart rate $\mathrm{HD}$ - hemodialysis

The all-cause mortality in the whole study group during the follow-up period was high, reaching $48 \%$. The diabetic HD patients showed higher mortality as compared to nondiabetic patients. The patients from this subgroup died more frequently due to non-cardiovascular causes.

Additionally, we found no significant difference in carotid stiffness parameters between the diabetic and non-diabetic patients after dividing the study population into subgroups by age: older and younger than 65 years. There has been no significant difference in echo-tracking parameters between diabetic and non-diabetic subjects with the aforementioned cardiovascular co-morbidities. We also found no significant difference between survivors and non-survivors in beta stiffness index, one-point pulse wave velocity, and epsilon both before and after hemodialysis as well as in arterial compliance before hemodialysis. Survivors and nonsurvivors differed significantly in arterial compliance after hemodialysis $(0.73$ [0.53-0.98] vs. 0.88 [0.72-1.33], $p=0.016)$. 
Table 4. Carotid stiffness parameters assessed by means of echo tracking between diabetic and non-diabetic patients

\begin{tabular}{|c|c|c|c|}
\hline & $\begin{array}{c}\text { Diabetic } \\
\mathrm{N}=37 \\
\text { median (IQR) }\end{array}$ & $\begin{array}{c}\text { Non-diabetic } \\
\mathrm{N}=53 \\
\text { median (IQR) }\end{array}$ & p \\
\hline$\beta$ before HD & $7.65[5.35-10.55]$ & $7.85[4.7-10.8]$ & 0.91 \\
\hline$\beta$ after HD & $7.7[5.3-9.0]$ & $7.1[5.35-8.65]$ & 0.53 \\
\hline$P W V \beta$ before $\mathrm{HD}[\mathrm{m} / \mathrm{s}]$ & $5.95[4.55-7.15]$ & $5.9[4.8-7.3]$ & 0.77 \\
\hline$P W V \beta$ after HD [m/s] & $5.8[4.7-6.5]$ & 5.8 [5.100-6.425] & 0.92 \\
\hline $\mathrm{AC}$ before $\mathrm{HD}[\mathrm{mm} 2 / \mathrm{kPa}]$ & $0.71[0.56-1.24]$ & $0.795[0.530-1.020]$ & 0.49 \\
\hline AC after $\mathrm{HD}[\mathrm{mm} 2 / \mathrm{kPa}]$ & $0.87[0.68-1.04]$ & $0.765[0.570-1.000]$ & 0.29 \\
\hline Ep before $\mathrm{HD}[\mathrm{kPa}]$ & $103[67-146]$ & 116 [69-155] & 0.76 \\
\hline Ep after $\mathrm{HD}[\mathrm{kPa}]$ & 99 [68-139] & 95 [72-119] & 0.64 \\
\hline
\end{tabular}

HD - hemodialysis; $\beta$ - beta stiffness index; PWV $\beta$ - one-point pulse wave velocity;

AC - arterial compliance; Ep - epsilon

Table 5. Cardiovascular and non-cardiovascular mortality - comparison in both groups

\begin{tabular}{cccc}
\hline & $\begin{array}{c}\text { Survived } \\
\mathbf{N}=\mathbf{4 7} \text { pts }\end{array}$ & Cardiovascular death $\mathbf{N}=\mathbf{1 9}$ pts & Non-cardiovascular death $\mathbf{N}=\mathbf{2 4}$ pts \\
\hline Non-diabetic patients & $74.47 \%$ & $52.63 \%$ & $33.33 \%$ \\
$\mathrm{~N}=53$ & $(35)$ & $(10)$ & $(8)$ \\
Diabetic patients & $25.53 \%$ & $47.37 \%$ & $66.67 \%$ * \\
$\mathrm{N}=37$ & $(12)$ & $(9)$ & $(16)$
\end{tabular}

${ }^{*} p<0.01$

\section{DISCUSSION}

There is growing evidence concerning the effect of diabetes mellitus on elastic properties of the arteries. It has been proven that diabetic patients are affected by higher arterial stiffness than non-diabetic subjects in the general population [17], in chronic kidney disease [12], and in end-stage renal disease [18]. In the available studies the arterial stiffness has been recognized as a useful tool in predicting developing cardiovascular disease [19] in diabetic patients and as a cardiovascular risk factor [10]. While arterial rigidity as a marker of so-called vascular aging is a well-established pathophysiological mechanism leading to cardiovascular diseases, there is still a discussion on the specifics of this process and on assessing arterial stiffness. The architecture of the arterial tree distinguishes elastic and muscular arteries that differ remarkably in wall structure, leading to physiological growth of stiffness in the distal vessels. Large elastic vessels such as aortas and carotid arteries play a key role in the cushioning function of the arterial system by reducing left ventricle afterload and by allowing non-pulsatile blood flow into the microcirculation [8, 20, 21]. Furthermore, in diabetes mellitus central arteries stiffen more significantly than peripheral ones, regardless of their renal function.
Pronounced stiffening of central arteries over peripheral arteries has been proven in diabetic patients with serum creatinine levels below $1.5 \mathrm{mg} / \mathrm{dl}$ [17] and with the progression of CKD [22]. This is also the case in end-stage renal disease patients on hemodialysis [23].

The discrepancy in the stiffening of different arteries indicates that the proximal aorta would be the preferred site to assess arterial stiffness. Unfortunately, no commonly used method can estimate stiffness of the ascending aorta, which leads to a search for a perfect surrogate. Most of performed studies have used pulse wave velocity as the reference method to assess arterial stiffness. It is still considered the gold standard by the European Cardiac Society [6] and, more recently, in the guidelines by the American Heart Association [24]. There is no recommendation in either document to use high-resolution echo-tracking as a method of arterial stiffness assessment in everyday clinical practice. Expert consensus by ESC states that local artery stiffness assessment methods are indicated for pathophysiological, pharmacological, and therapeutic studies.

Our results indicate no further influence of diabetes on carotid stiffness parameters assessed by the echo-tracking method in hemodialyzed patients. This contradicts the observations of Sato et al. where the presence of diabetes 
mellitus was independently associated with the beta stiffness parameter [14]. Other studies on HD patients have shown a connection between an elevated beta stiffness parameter and the presence of diabetes $[15,16]$. Although in the study Wang et al. conducted in patients with CKD, just GFR estimated per $1.73 \mathrm{~m}^{2}$ and systolic BP - but not diabetes mellitus - were the major clinical determinants of arterial stiffness in a multivariate model [25]. The difference between our results and the other observations could result from the baseline characteristics of the study group, such as age or the duration and cause of chronic kidney disease. It should be taken into account that in a population with multiple factors contributing to an increase in arterial stiffness, a single factor may no longer be of significant importance when these changes are advanced. Thus, if we were studying a younger population [12,18], the presence of diabetes could have a significant impact on the progression of arterial stiffness; in our population, about a decade older, this effect may not be as obvious. The comparison of carotid stiffness parameters between survivors and non-survivors in the whole study group has shown no relation between aggravated arterial stiffness and survival. On the contrary, the deceased patients had increased AC after hemodialysis, a marker of high arterial elasticity, which suggests paradoxical and complex mechanisms of vascular function in this population.

After analyzing subgroups of patients with highest cardiovascular risk (history of one or more of the following: coronary artery disease, myocardial infarction, coronary artery bypass graft, percutaneous coronary intervention, or stroke) and lowest cardiovascular risk, we did not observe a statistically significant difference in carotid stiffness, which is probably due to the fact that hemodialyzed patients with no history of cardiovascular disease still suffer from high mortality risk attributable only to chronic kidney disease and/ or its treatment. In our study we have also tried to assess the role of age in the utility of echo-tracking as a diagnostic method. After dividing the study population into subjects over and under 65 years old, we found no statistically significant differences between the diabetic and non-diabetic patients in carotid stiffness parameters. Furthermore, our findings may correspond with the results of the study by Paini et al. [26] which have shown a difference in progression of aortic and carotid stiffness between groups of patients with diabetes mellitus type 2 and/or hypertension, and healthy subjects, showing that the common carotid artery stiffens less prominently than the aorta in patients with increased cardiovascular risk.

Last but not least in our study, in a relatively long 58-month follow-up we observed a significant role of diabetes mellitus on general mortality, which was higher in the diabetic group. However, we found that diabetes is more common in subjects who suffer from non-cardiovascular death rather than from cardiovascular death. This may be due to septic complications in the diabetic group. The problem of the complexity of the phenomenon of increased mortality in patients with CKD was perfectly reflected in the work of $\mathrm{K}$. Jager et al. The authors pointed out that patients in this population die more often than subjects at comparable age also due to non-cardiovascular causes; for example, because the risk of sepsis in dialysis patients is 50 times higher than in the general population [27].

\section{CONCLUSIONS}

1. High-resolution echo-tracking might be considered a valuable arterial stiffness assessment method; however, its utility in high cardiovascular risk groups such as patients on maintenance hemodialysis and diabetes may be limited.

2. High-resolution echo-tracking parameters in hemodialyzed patients are not influenced by the presence of diabetes.

3. The presence of diabetes in hemodialyzed patients, according to our results, paradoxically does not add an additional risk factor in this high cardiovascular risk group.

\section{STRENGTHS AND LIMITATIONS}

Our study has several limitations. The most important one is the observational nature of the investigation, so causality can't be directly derived from the results. The protocol of this study assumed an assessment of local arterial stiffness, baseline characteristics, and laboratory parameters at the beginning of the study, and performed an analysis of the survival of patients after completion of the follow-up. It had also a relatively small number of participants. On the other hand, one of the strengths of our study was measurement of high-resolution echo-tracking parameters before and after hemodialysis and the 58-month-long follow-up. As the subject did not undergo extensive investigations, our study adds an important piece of information regarding the studied issue.

\section{Abbreviations}

ACEI - angiotensin converting enzyme inhibitors; AGEs - advanced glycation end products; ARBs - angiotensin receptor blockers; BMI - body mass index; DBP - diastolic blood pressure; ESAs - erythropoiesis stimulating agents; HD - hemodialysis, HR - heart rate, $\mathbf{K t} / \mathbf{V}$ - adequacy of dialysis, SBP - systolic blood pressure. 


\section{Authors' Contribution}

K.R.: research concept and design, acquisition of data, data analysis and interpretation, writing - original draft preparation, literature review; A.S.: acquisition of data, data analysis and interpretation, literature review; M.O.: acquisition of data, literature review, writing - review and editing; K.M.: research concept and design, supervising the project, data analysis and interpretation, final proofreading, and approval of the version.

\section{Conflict of Interest}

The authors have no potential conflicts of interest to declare.

\section{Ethics Approval}

The consent was issued by the Bioethics Committee at the Medical University of Wrocław (No. KB 342/2017).

\section{References}

[1] Fox C.S., Matsushita K., Woodward M., Bilo H.J., Chalmers J., Heerspink H.J., Lee B.J., Perkins R.M., Rossing P., Sairenchi T., et al. (2012). Chronic Kidney Disease Prognosis Consortium. Associations of kidney disease measures with mortality and end-stage renal disease in individuals with and without diabetes: a metaanalysis. Lancet, 380: 1662-1673.

[2] Tuttle K.R., Bakris G.L., Bilous R.W., Chiang J.L., de Boer I.H., Goldstein-Fuchs J., Hirsch I.B., Kalantar-Zadeh K., Narva A.S., Navaneethan S.D., et al. (2014). Diabetic kidney disease: A report from an ADA Consensus Conference. Diabetes Care, 37: 2864-2883.

[3] Saran R., Robinson B., Abbott K.C., Agodoa L.Y., Bragg-Gresham J., Balkrishnan R., Bhave N., Dietrich X., Ding Z., Eggers P.W., et al. (2019). US Renal Data System 2018 Annual Data Report: Epidemiology of kidney disease in the United States. Am. J. Kidney Dis., 73: A7-A8.

[4] Karras A., Haymann J.P., Bozec E., Metzger M., Jacquot C., Maruani G., Houillier P., Froissart M., Stengel B., Guardiola P., et al. (2012). Nephro Test Study Group. Large artery stiffening and remodeling are independently associated with all-cause mortality and cardiovascular events in chronic kidney disease. Hypertension, 60: 1451-1457.

[5] Williams B., Mancia G., Spiering W., Rosei E.A., Azizi M., Burnier M., Clement D.L., Coca A., de Simone G., Dominiczak A., et al. (2019). 2018 ESC/ESH Guidelines for the management of arterial hypertension. Kardiol. Pol., 77: 71-159
[6] Laurent S., Cockcroft J., Van Bortel L., Boutouyrie P., Giannattasio C., Hayoz D., Pannier B., Vlachopoulos C., Wilkinson I., StruijkerBoudier H. (2006). European Network for Non-invasive Investigation of Large Arteries: Expert consensus document on arterial stiffness: Methodological issues and clinical applications. Eur. Heart J., 27: 2588-2605.

[7] DeLoach S.S., Townsend R.R. (2008). Vascular stiffness: Its measurement and significance for epidemiologic and outcome studies. Clin. J. Am. Soc. Nephrol., 3: 184-192.

[8] London G.M. (2018). Arterial stiffness in chronic kidney disease and end-stage renal disease. Blood Purif., 45: 154-158.

[9] Mattace-Raso F.U., van der Cammen T.J., Hofman A., van Popele N.M., Bos M.L., Schalekamp M.A., Asmar R., Reneman R.S., Hoeks A.P., Breteler M.M., Witteman J.C. (2006). Arterial stiffness and risk of coronary heart disease and stroke: The Rotterdam Study. Circulation, 113: 657-663.

[10] Shoji T., Emoto M., Shinohara K., Kakiya R., Tsujimoto Y., Kishimoto H., Ishimura E., Tabata T., Nishizawa Y. (2001). Diabetes mellitus, aortic stiffness, and cardiovascular mortality in end-stage renal disease. J. Am. Soc. Nephrol., 12: 2117-2124.

[11] Zanoli L., Lentini P., Briet M., Castellino P., House A.A., London G.M., Malatino L., McCullough P.A., Mikhailidis D.P., Boutouyrie P. (2019). Arterial stiffness in the heart disease of CKD. J. Am. Soc. Nephrol., 30: 918-928.

[12] Stróżecki P., Kurowski R., Flisiński M., Stefańska A., OdrowążSypniewska G., Manitius J. (2013). Advanced glycation end products and arterial stiffness in patients with diabetic nephropathy and patients with chronic kidney disease without diabetes. Pol. Arch. Med. Wewn., 123: 609-616.

[13] Zieman S.J., Melenovsky V., Kass D.A. (2005). Mechanisms, pathophysiology, and therapy of arterial stiffness. Arterioscler. Thromb. Vasc. Biol., 25: 932-943.

[14] Sato M., Ogawa T., Otsuka K., Ando Y., Nitta K. (2013). Stiffness parameter $\beta$ as a predictor of the 4-year all-cause mortality of chronic hemodialysis patients. Clin. Exp. Nephrol., 17: 268-274.

[15] Shoji T., Maekawa K., Emoto M., Okuno S., Yamakawa T., Ishimura E., Inaba M., Nishizawa Y. (2010). Arterial stiffness predicts cardiovascular death independent of arterial thickness in a cohort of hemodialysis patients. Atherosclerosis, 210: 145-149.

[16] Yu Z.X., Wang X.Z., Guo R.J., Zhong Z.X., Zhou Y.L. (2013). Comparison of ultrasound echo-tracking technology and pulse wave velocity for measuring carotid elasticity among hemodialysis patients. Hemodial. Int., 17: 19-23.

[17] Kimoto E., Shoji T., Shinohara K., Inaba M., Okuno Y., Miki T., Koyama H., Emoto M., Nishizawa Y. (2003). Preferential stiffening of central over peripheral arteries in type 2 diabetes. Diabetes, 52 : 448-452.

[18] Kim E.Y., Yi J.H., Han S.W., Shin J., Lee J.U., Kim S.G., Kim H.J. (2008). Clinical factors associated with brachial-ankle pulse wave velocity in patients on maintenance hemodialysis. Electrolyte Blood Press, 6: 61-67. 
[19] Ohkuma T., Ninomiya T., Tomiyama H., Kario K., Hoshide S., Kita Y., Inoguchi T., Maeda Y., Kohara K., Tabara Y., et al. (2017). Brachial-ankle pulse wave velocity and the risk prediction of cardiovascular disease: An individual participant data meta-analysis. Hypertension, 69: 1045-1052.

[20] Briet M., Boutouyrie P., Laurent S., London G.M. (2012). Arterial stiffness and pulse pressure in CKD and ESRD. Kidney Int., 82: 388-400.

[21] Milan A., Tosello F., Fabbri A., Vairo A., Leone D., Chiarlo M., Covella M., Veglio F. (2011). Arterial stiffness: From physiology to clinical implications. High Blood Press. Cardiovasc. Prev., 18: $1-12$.

[22] Kimoto E., Shoji T., Shinohara K., Hatsuda S., Mori K., Fukumoto S., Koyama H., Emoto M., Okuno Y., Nishizawa Y. (2006). Regional arterial stiffness in patients with type 2 diabetes and chronic kidney disease. J. Am. Soc. Nephrol., 17: 2245-2252.

[23] London G.M., Marchais S.J., Safar M.E., Genest A.F., Guerin A.P., Metivier F., Chedid K., London A.M. (1990). Aortic and large artery compliance in end-stage renal failure. Kidney Int., 37: 137-142.
[24] Townsend R.R.., Wilkinson I.B., Schiffrin E.L., Avolio A.P., Chirinos J.A., Cockcroft J.R., Heffernan K.S., Lakatta E.G., McEniery C.M., Mitchell G.F., et al. (2015). Recommendations for improving and standardizing vascular research on arterial stiffness: A scientific statement from the American Heart Association. Hypertension, 66: 698-722.

[25] Wang M.C., Tsai W.C., Chen J.Y., Huang J.J. (2005). Stepwise increase in arterial stiffness corresponding with the stages of chronic kidney disease. Am. J. Kidney Dis., 45: 494-501.

[26] Paini A., Boutouyrie P., Calvet D., Tropeano A.I., Laloux B., Laurent S.: Carotid and aortic stiffness (2006). Determinants of discrepancies. Hypertension, 47: 371-376.

[27] Jager K.J., Lindholm B., Goldsmith D., Fliser D., Wiecek A., Suleymanlar G., Ortiz A., Massy Z., Martinez-Castelao A., Agarwal R., et al. (2001). Cardiovascular and non-cardiovascular mortality in dialysis patients: Where is the link? Kidney Int. Suppl., 1: 21-23. 